\title{
SURFACE PROPERTIES OF RESIN-INFILTRATED INCIPIENT ENAMEL LESIONS AFTER AGING UNDER THERMAL STRESSES
}

\author{
Enas T. Enan*, Marwa A. Tawfik**, Ashraf I. Ali*** and Ahmed T. Anan ${ }^{* * * *}$
}

\begin{abstract}
Introduction: Resin infiltration technique is a promising option for treatment of incipient enamel lesions, especially for patients with history of fixed orthodontic appliances. However, its ability to resist aging under thermal influences in the oral cavity is still questionable. Aim of the study: The present research aimed to inspect the ability of resin-infiltrated initial enamel lesions, to resist aging under thermal challenge.

Materials and methods: Fifty extracted non-carious premolars were included in the present study. Specimens were equally distributed into 5 groups, where group 1 included teeth with sound enamel (control), while group 2 included teeth with decalcified untreated lesions. Specimens of group 3 were subjected to Icon ${ }^{\circledR}$ resin infiltration after decalcification. For groups 4 and 5 , decalcified resin-infiltrated specimens were subjected to thermocycling (TC) at 5,000 or 10,000 cycles respectively. Surface evaluation parameters included surface microhardness and roughness. Surface morphology was further evaluated using scanning electron microscope (SEM). For statistical analysis, ANOVA and LSD tests were used.
\end{abstract}

Results: Resin-infiltrated enamel was more resistant to surface changes, under thermal stresses, than the non-resin-infiltrated enamel. The resin surface showed high resistance to surface degradation at thermal stress of 5000 cycles, while more deterioration started to appear at 10,000 cycles.

Conclusion: Resin infiltration has the ability to provide adequate protection to the demineralized enamel against thermal attack of 5,000 thermal cycles. However, surface microcracks generated at 10,000 thermal cycles indicate that the material might further deteriorate on aging for more than one clinical service year.

KEYWORDS: Incipient enamel lesions, Resin infiltration, Thermocycling.

\footnotetext{
* Associate professor, Department of Dental Biomaterials, Faculty of Dentistry, Mansoura University, Mansoura, Egypt.

** Lecturer, Department of Orthodontics, Faculty of Dentistry, Mansoura University, Mansoura, Egypt.

*** Lecturer, Department of operative Dentistry, Faculty of Dentistry, Mansoura University, Mansoura, Egypt.

**** BDS, MPH, NBDE Dental practitioner, Mansoura Health Insurance Institute, Mansoura, Egypt.
} 


\section{INTRODUCTION}

Caries develops as a result of dynamic periods of demineralization and remineralization. ${ }^{1}$ Demineralization takes place when enamel is invaded by pathogenic bacteria that produce organic acids, by fermentation of food debris. Subsequently, calcium and phosphate minerals are dissolved out of the enamel, by the action of acids, leaving microporosities within the remaining enamel. If the dissolved minerals are not compensated for by successive remineralization process, this leads to more caries progression., ${ }^{2} 3$ This process is more pronounced in patients who are unable to maintain adequate oral hygiene, such as handicapped children or patients with fixed orthodontic appliances. ${ }^{4}$

Formerly, the most commonly used strategy for treatment of incipient enamel caries included enhancing enamel remineralization by using calcium phosphate or fluoride-containing products. Several reminerlizing products were introduced to the markets since the year 2000, where their mechanism of action was based on enhancing the natural reminiralization property of saliva.-7 Although this technique could be helpful to some patients, yet, it was reported by some studies that it is not generally recommended for all cases as it doesn't provide a general solution for controlling dental caries. ${ }^{8,9}$

In year 2009, Icon ${ }^{\circledR}$ product for caries infiltration, was introduced in Germany, as an innovative option for micro-invasive treatment of early carious lesions without drilling. ${ }^{10}$ This technique applies a low-viscosity light-curing unfilled resin composed of triethylene glycol dimethacrylate (TEGDMA). The high fluidity of this unfilled resin enables it to easily infiltrate into the caries-produced porosity, and thus, occluding the diffusion pathways of caries producing acids. By this painless, non-drilling technique, the resin can arrest progression of caries with no need for unnecessary loss of healthy dental tissue. ${ }^{7,11}$
Several studies were conducted to test Icon resin infiltrant, and most of them have shown promising results. ${ }^{12-16}$ However, concerns are still there regarding its ability to provide a longterm protection against cyclic changes caused by different oral conditions. Therefore, the present research aimed to evaluate the effect of aging under thermal cycles, on surface hardness and roughness of Icon-treated incipient enamel lesions. Our null hypothesis assumed that there were no statistically significant differences between the tested groups with thermocycling.

\section{MATERIALS AND METHODS}

\section{Specimens' preparation and grouping}

Fifty non-carious upper and lower bicuspids, free from cracks and defects, obtained from orthodontic clinic, were cleaned and kept at room temperature in $0.1 \%$ thymol. Roots were removed away with a low speed diamond disc (Isomet ${ }^{\mathrm{TM}}$, Buehler, Lake Bluff, IL). Specimens were embedded in acryl resin blocks leaving the relatively flat proximal surface uncovered for evaluation.

Then, the prepared specimens $(n=50)$ were randomly divided into 5 groups (10 specimens each) as follows:

1. Sound enamel (control).

2. Untreated lesion.

3. Resin-treated lesion.

4. Resin-treated lesion + thermocycled at 5000 cycles.

5. Resin-treated lesion + thermocycled at 10000 cycles.

\section{Creation of incipient enamel lesions}

Before embedding in acrylic blocks, enamel lesions were artificially created on specimens of 
groups 2, 3, 4 and 5 using an acidic demineralizing solution (50 $\mathrm{mM}$ acetic acid, $3 \mathrm{mM} \mathrm{KH} 2 \mathrm{PO} 4,3 \mathrm{mM}$ $\mathrm{CaCl} 2$ H2O, 6m M methylhydroxydiphosphonate, $\mathrm{pH} 4.95,37^{\circ} \mathrm{C}$ ) as described by Subramaniam et al. ${ }^{17}$

Specimens were immersed in the demineralizing solution for $160 \mathrm{~h}$, to create incipient lesions on enamel surface. ${ }^{18}$ Throughout the decalcification procedures, $\mathrm{pH}$ was checked and adjusted using $10 \% \mathrm{HCl}$ or $10 \mathrm{M} \mathrm{KOH}$ when needed. The amount of demineralization was examined under microscope to replace specimens with inadequate enamel lesion.

\section{Application of resin infiltrant}

Specimens were coated with resin infiltrant (IconVR, DMG, Hamburg, Germany) following the guidelines of manufacturer:

1. Acid etching, using $15 \%$ hydrochloric acid gel

(IconVR -Etch, DMG, Hamburg, Germany) for $2 \mathrm{~min}$.

2. Thorough washing and drying for $30 \mathrm{~s}$.

3. Ethanol application (IconVR -Dry, DMG, Hamburg,Germany) and drying.

4. Infiltrant application, removal of residual resin, then curing with conventional halogen light (3 M Curing Light 2500, $3 \mathrm{M}$, USA) for $40 \mathrm{~s}$.

5. Re-application and curing of resin infiltrant.

\section{Thermocycling (TC)}

For the thermocycling groups, a thermocycling machine (THE-1100 thermocycler, SD Mechatronik, Feldkirchen-Westerham, Deutschland) was used. The thermocycler was programmed to perform 5000 cycles (group 4) or 10,000 cycles (group 5) between two water baths at temperatures of $5^{\circ} \mathrm{C}$ and $55^{\circ} \mathrm{C}$, respectively, with a dwell time of 30 seconds at each temperature. ${ }^{19}$

\section{Surface microhardness evaluation}

Microhardness of the tested specimens was measured using Shimadzu microhardness tester (HMV-2T, Shimadzu Corporation, Kyoto, Japan) with a Vickers elongated diamond pyramid indenter. The tested surface was subjected to a constant load of $50 \mathrm{~g}$ for $10 \mathrm{~s}$ dwell time. Three indentations were made on the center of tooth surface and the mean value considered as the Vickers Hardness Number (VHN). ${ }^{20}$

\section{Evaluation of surface roughness and morphology}

A digital profilometer (Mitutoyo Surf Test SJ 210 Analyzer; Mitutoyo Corp, Japan) was used to record surface roughness ( $\mathrm{Ra}$ ) values for all the specimens. During measurement of Ra, a constant load of $3.9 \mathrm{~mm}$ was applied to the diamond stylus which traveled across the enamel surface at a speed of $0.5 \mathrm{~mm} / \mathrm{s}$ and a tracing distance of $8 \mathrm{~mm}$. The instrument calibration was made with a standard reference specimen. The resolution of the recorded data was $0.01 \mu \mathrm{m}$. The same procedure was conducted for 3 times per specimen and the average was determined as the mean roughness parameter (Ra) value $(\mu \mathrm{m})$.

For further evaluation of surface morphology, specimens from each group were randomly selected and inspected with Scanning Electron Microscope (SEM) (JEOL JSM-5200, Japan). The selected specimens were firstly gold sputtered for better image resolution, and then analyzed, at a magnification of $2000 \mathrm{X}$.

\section{Statistical analysis}

Statistical analysis software (SPSS 12.0, SPSS, Chicago, USA) was used to analyze the gathered data. Data were further tested by one-way analysis of variance (ANOVA) and LSD post hoc tests for individual comparisons between groups, at a significance level of $5 \%$. 


\section{RESULTS}

\section{Surface microhardness}

Table 1 shows means and standard deviations for VHN results. The sound enamel group displayed the highest VHN value (244.28), and the group of untreated lesion showed the lowest value (103.96). One way ANOVA test proofed a significant difference among the tested groups $(\mathrm{p}<.0001)$. LSD test showed significant difference between all resintreated groups (3,4 and 5) and the sound enamel (control) group $(\mathrm{p}<.0001)$. Comparing the thermocycled groups (4 and 5) to the resin-treated group without thermocycling (group 3), showed that the 10000 cycles (group 5) caused significant decrease in VHN $(\mathrm{p}<.0001)$, while the 5000 cycles (group 4) caused an insignificant difference. However, the surface microhardness of both thermo-cycled resin-treated specimens (groups 4 and 5) were significantly higher than that of the untreated lesion (group 2).

\section{Surface roughness and topography}

Table 2 represents means and standard deviations collected from the Ra test. The highest $\mathrm{Ra}$ mean value was recorded for group 2 of the untreated lesion $(1.88 \mu \mathrm{m})$, while group 3 of the resin-treated specimens showed the lowest value $(0.53 \mu \mathrm{m})$. One way ANOVA test indicated a significant difference among the tested groups $(\mathrm{p}<.0001)$.

LSD test proofed significant difference between all resin-treated groups (3, 4 and 5) and the group 2 (untreated lesion) $(\mathrm{p}<.0001)$. Significant difference was also found between group 1 (control) and group 5, while an insignificant difference was found between group 1 and each of groups 3 and 4. Comparing the three resin-treated groups $(3,4$

TABLE (1) VHN results for all groups

\begin{tabular}{|c|c|c|c|c|}
\hline Groups & Mean \pm Sd & F- value & $\mathrm{P}$ - value & LSD \\
\hline 1. Sound enamel (control) & $244.28^{\mathrm{A}} \pm 4.60$ & \multirow{5}{*}{132.96} & \multirow{5}{*}{$<.0001$} & \multirow{5}{*}{13.48} \\
\hline 2. Untreated lesion & $103.96^{\mathrm{D}} \pm 7.23$ & & & \\
\hline 3. Resin-treated & $214.96^{\mathrm{B}} \pm 16.07$ & & & \\
\hline 4. Resin-treated + TC 5000 & $201.76^{\text {в С }} \pm 9.53$ & & & \\
\hline 5. Resin-treated + TC 10000 & $190.64^{\mathrm{C}} \pm 9.97$ & & & \\
\hline
\end{tabular}

Means with the same superscript letter are not significantly different at $P \leq 0.05$

TABLE (2) Ra results for all groups ( $\mu \mathrm{m})$.

\begin{tabular}{|c|c|c|c|c|}
\hline Groups & Mean $\pm \mathrm{Sd}$ & F-value & P-value & LSD \\
\hline 1. Sound enamel (control) & $0.78^{\mathrm{C}} \pm 0.096$ & \multirow[t]{5}{*}{38.56} & \multirow[t]{5}{*}{$<.0001$} & \multirow[t]{5}{*}{0.2587} \\
\hline 2. Untreated lesion & $1.88^{\mathrm{A}} \pm 0.34$ & & & \\
\hline 3. Resin-treated & $0.53^{\mathrm{C}} \pm 0.11$ & & & \\
\hline 4. Resin-treated + TC 5000 & $0.62^{\mathrm{C}} \pm 0.088$ & & & \\
\hline 5. Resin-treated + TC 10000 & $1.07^{\mathrm{B}} \pm 0.22$ & & & \\
\hline
\end{tabular}


and 5) to the untreated lesion revealed significant differences.

Scanning electron micrographs of the tested groups are shown in Figure 1. In Figure 1.1, the characteristic fish scales appearance of healthy enamel can be obviously seen on the surface of control specimen. While in Figure 1.2 the specimen with untreated enamel lesion showed a sponge-like
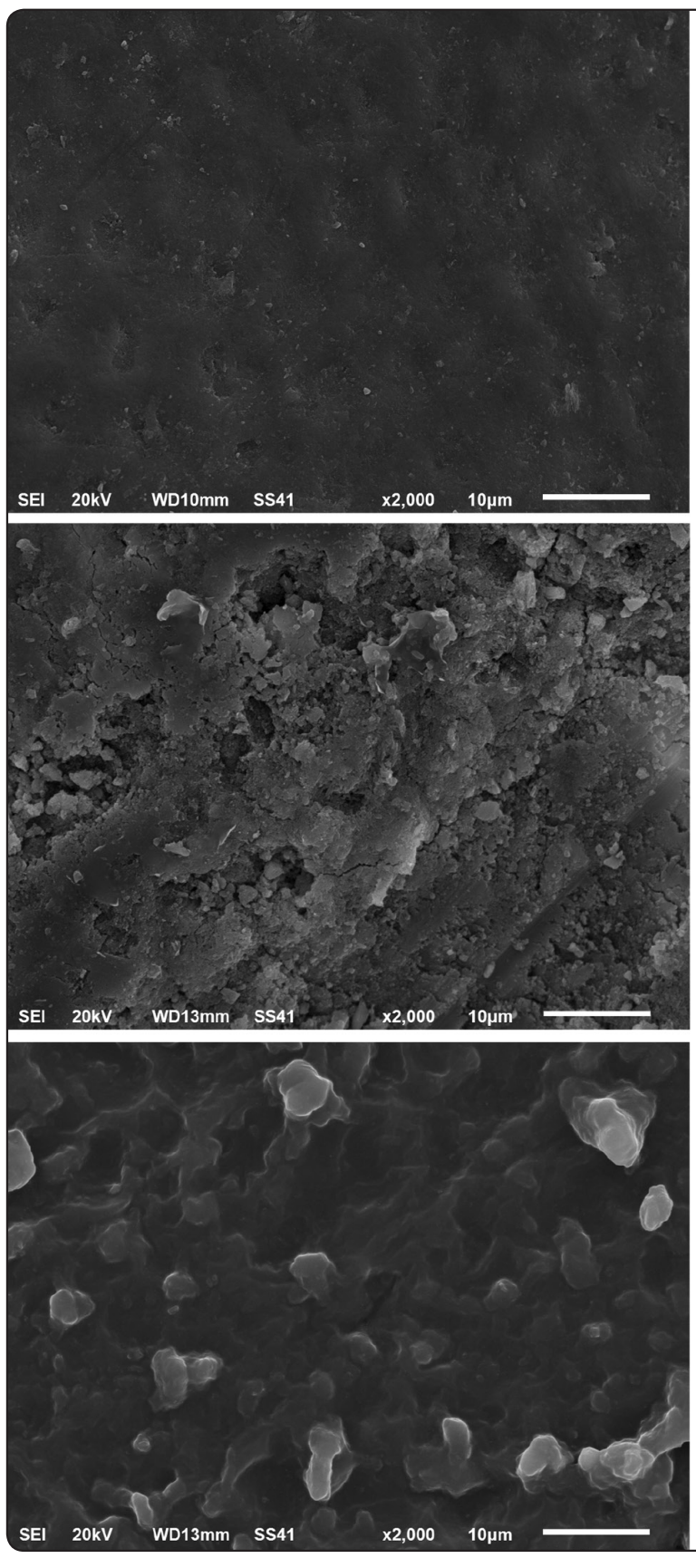

5. Resin-treated lesion + thermocycled at 10000 cycles.
Fig. (1) SE micrographs (2000 X) showing enamel surface of all groups:

1. Sound enamel.

2. Untreated lesion.

3. Resin-treated lesion.

4. Resin-treated lesion + thermocycled at 5000 cycles.

structure and severe enamel erosion. SE micrographs of the resin-treated specimen in Figure 1.3 showed less surface irregularities and smoother surface. Figure 1.4 showed some surface porosity which is still less extensive than that of the untreated lesion. In Figure 1.5 surface microcracks and microfissures could be observed on the resin-infiltrated lesions after exposure to 10,000 thermal cycles.
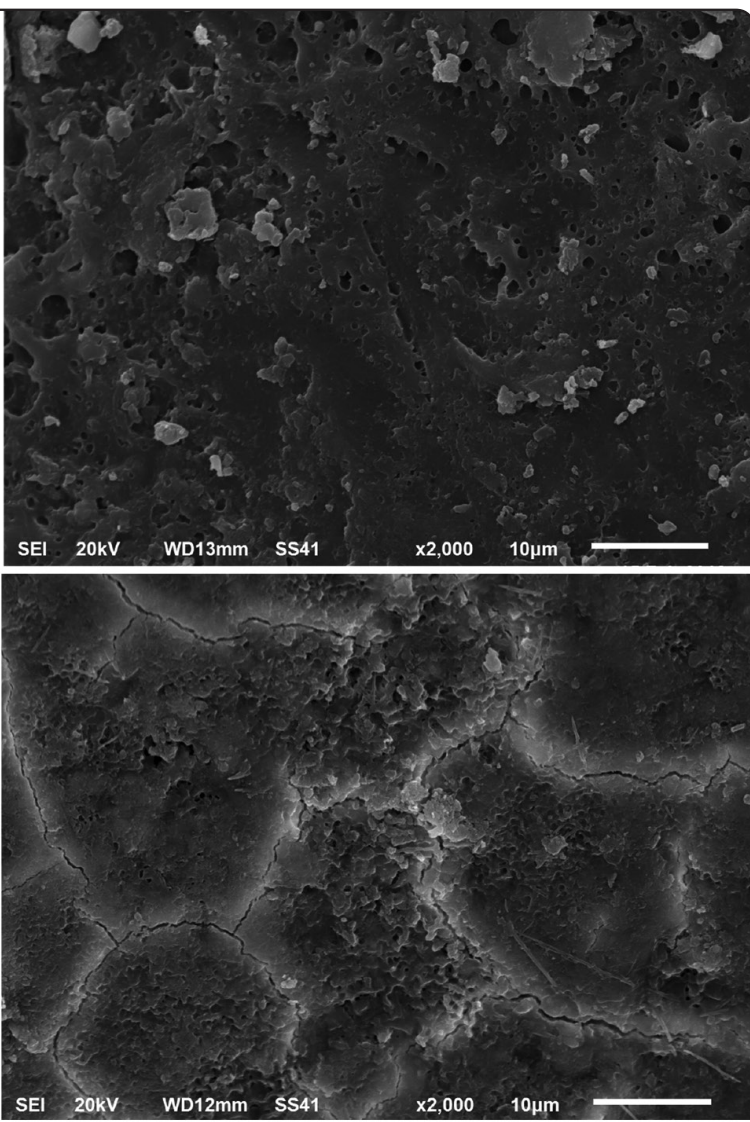


\section{DISCUSSION}

Resin infiltration technique gained a good reputation as a successful option for treatment of incipient enamel lesions, especially for patients with history of fixed orthodontic appliances. ${ }^{12,21}$ Nevertheless, its ability to resist aging and degradation by different factors in the oral cavity is still questionable. ${ }^{22}$

Thus, the current research was conducted to estimate the capability of Icon resin infilrant to resist degradation under the effect of thermal aging. For this purpose, TC was conducted at 5,000 and 10,000 cycles respectively. It was stated by previous studies that 5000 cycles of TC equal about half year in service, while 10,000 cycles may be equal to one year in clinical service..$^{23,24}$ Surface microhardness and roughness were selected as parameters for evaluation in this study because changes in these properties may contribute to more problems such as staining and discoloration of resin-infiltrated surfaces, plaque accumulation with subsequent secondary caries formation or even failure of restoration. $^{25}$

Findings of the present study indicated a positive effect of Icon resin on surface hardness of decalcified enamel lesions, as the specimens with Icon resin showed significantly higher VHN values in comparison to specimens with untreated lesions. Regarding the effect of thermocycling on Icon surface, our results showed that 5000 thermal cycles did not cause significant effect, while TC at 10000 cycles caused a significant reduction in surface hardness of the resin-treated specimens. However, even with TC at 10000 cycles, VHN of resin-treated specimens was still significantly higher than that of specimens with untreated lesion. The findings of the current research came in agreement with those reported by El-zankalouny et al. ${ }^{4}$, Torres et al. ${ }^{26}$ and Paris et al. ${ }^{27}$ in that the microhardness of carious lesions was significantly increased with resin infiltration when compared with untreated artificial lesions.
The ability of Icon resin to recover enamel microhardness can be explained by the low viscosity of the resin, which enables it to penetrate deeply into enamel lesions immediately after treatment. It seems that the infiltration resin has the ability to encapsulate the remaining hydroxyapatite crystals at the site of enamel lesion, giving a uniform resinhydroxyapatite complex which possessed a high surface hardness.

Alternating temperature fluctuations in the oral environment may enhance bond degradation between the infiltrating resin and enamel hydoxyapatite because of differences in coefficients of thermal expansion between them. ${ }^{28,29}$

Regarding surface roughness, our results indicated that resin infiltration could provide smoother enamel surface, as compared to untreated lesions. On exposure to thermal challenge, Icontreated specimens could maintain smoother surface, both at 5000 and 10,000 cycles. Further comparison of the thermocycled groups revealed that Icontreated specimens with TC at 5000 cycles did not show a significant difference from that of the sound enamel. On the othe hand, 10,000 TC cycles caused a significantly higher surface roughness of Icontreated enamel, when compared to sound enamel surface. This may indicate that Icon starts showing some sort of deterioration at this number of thermal cycles. The increased surface roughness observed on the resin-infiltrated lesions with aging in the present study, may be attributed to internal and thermal stresses generated by water sorption and thermal expansion and contraction. ${ }^{19}$

The present results agreed with former studies ${ }^{30-32}$ which reported the ability of resin infiltrants to provide and maintain smoother enamel surface. However, other studies ${ }^{19,33,34}$ came in contrast, as they reported that surface roughness of resin-treated enamel lesions were not ideal and might further recede with aging. This contrast can be explained by mismatching in the research conditions and methods of evaluation. 


\section{CONCLUSION AND RECOMMENDATION}

Under conditions of the current study, it could be reported that the Icon resin infiltration could effectively improve and maintain surface hardness and roughness of demineralized enamel lesions, even after aging under thermal challenge of 5000 thermal cycles. Nevertheless, at 10,000 thermal cycles, surface microcracks and microfissures started to generate on the surface of resin-infiltrated lesions, which indicates that the material might further deteriorate after aging in the oral environment for more than one year. Further studies are needed to evaluate its long term durability aginst different factors encountered in the oral cavity, and to verify the necessity for renewing the resin infiltration after one year in service, as represented by 10,000 thermal cycles .

\section{REFERENCES}

1. Cha SW, Yoon TC, Park SH, Lee CY, Kum KY. Quantitative analysis of mineral change in the initial carious lesion using confocal laser scanning microscopy. J Kor Acad Cons Dent. 2001; 26: 1-8.

2. Ten Cate JM., Larsen MJ, Pearce EIF, Fejerskov O. Chemical interactions between the tooth and oral fluids. In: Dental Caries. Blackwell Munksgaard, Oxford, 2003 pp. 49-69.

3. Kidd EA, Fejerskov O. What constitutes dental caries? Histopathology of carious enamel and dentin related to the action of cariogenic biofilms. J Dent Res. 2004; 83 : C35-38.

4. El-zankalouny SM, Abd El Fattah WM, El- Shabrawy SM. Penetration depth and enamel microhardness of resin infiltrant and traditional techniques for treatment of artificial enamel lesions. Alex Dent J 2016; 41: 20-25.

5. Kwun JW, Kim KY, Lee SJ, Jung IY, Lee CY. The effect of the supersaturated solutions containing high concentrations of fluoride on seeded crystal growth. J Kor Acad Cons Dent. 1999; 24: 330-6.

6. Paris, S., Meyer-Lueckel, H., Mueller, J., Hummel, M., Kielbassa, A.M. Progression of sealed initial bovine enamel lesions under demineralising conditions in vitro. Caries Res 2006; 40: 129-134.
7. Paris $\mathrm{S}$, Meyer-Lueckel H. Inhibition of caries progression by resin infiltration in situ. Caries Res 2010; 44: 47-54.

8. Cury JA, Tenuta, LM. Enamel remineralization: controlling the caries disease or treating early caries lesions? Braz. Oral Res 2009; 23: 23-30.

9. Reynolds, E.C. Calcium phosphate-based remineralization systems: scientific evidence? Aust Dent J 2008; 53 (3), 268-273.

10. Taher NM, Alkhamis HA, Dowaidi SM. The influence of resin infiltration system on enamel microhardness and surface roughness: An in vitro study. The Saudi Dental Journal 2012; 24: 79-84.

11. Mueller J, Meyer-Lueckel H, Paris S, Hopfenmuller W, Kielbassa AM. Inhibition of lesion progression by the penetration of resins in vitro: Influence of the application procedure. Oper Dent 2006; 31: 338- 45.

12. Paris S, Meyer-Lueckel H. Masking of labial enamel white spot lesions by resin infiltration: a clinical report. Quintessence Int 2009; 40: 173-178.

13. Kn“osel M, Eckstein A, Helms HJ. Durability of esthetic improvement following Icon resin infiltration of multi bracket induced white spot lesions compared with no therapy over 6 months: a single-center, split-mouth, randomized clinical trial. Am J Orthod Dentofac Orthop 2013; 144: 86-96.

14. Paris S, Schwendicke F, Keltsch J, D“ orfer C, Meyer-Lueckel H. Masking of white spot lesions by resin infiltration in vitro. J Dent 2013; 41: e28-e34.

15. Rey N, Benbachir N, Bortolotto T, Krejci I. Evaluation of the staining potential of a caries infiltrant in comparison to other products. Dent Mat J 2014; 33: 86-91.

16. Yuan H, Li J, Chen L, Cheng L, Cannon RD, Mei L. Esthetic comparison of white-spot lesion treatment modalities using spectrometry and fluorescence. Angle Orthod. 2014; 84: 343-349.

17. Subramaniam P, Babu KLG, Lakhotia D. Evaluation of penetration depth of a commercially available resin infiltrate into artificially created enamel lesions: an in vitro study. J Conserv Dent 2014; 17: 146-149.

18. Gelgor IE, Ercan E, Akyalcin S, Dulgergil T, Catalbas B. A scaning electron microscope investigation into white spot lesion removal with microabrasion approach. Turkiye Klinikleri J Dental Sci. 2009; 15: 11-17. 
19. Zhao X, Ren Y-F. Surface Properties and Color Stability of Resin-Infiltrated Enamel Lesions. Operative Dentistry 2016; 41: 617-626.

20. Davari AR, Kazemi AD, Ataei E, Vatanpour M, Abdollahi $\mathrm{H}$. Effects of bleaching and remineralizing agents on the surface hardness of enamel. J Dent. 2012; 13: 156-63.

21. Meyer-Lueckel H, Paris S. Progression of artificial enamel caries lesions after infiltration with experimental light curing resins. Caries Res 2008; 42: 117-124.

22. Torres CRG, Borges AB, Torres LMS, Gomes IS, De Oliveira RS. Effect of caries infiltration technique and fluoride therapy on the colour masking of white spot lesions. J Dent 2011; 39: 202-207.

23. Gale M, Darvell B. Thermal cycling procedures for laboratory testing of dental restorations. J Dent 1999; 27: 89-99.

24. Yap AUJ, Wang X, Wu X, Chung SM. Comparative hardness and modulus of tooth-colored restoratives: A depthsensing microindentation study. Biomaterials 2004; 25 : 2179-2185.

25. Borges A, Caneppele T, Luz M, Pucci C, \& Torres C. Color stability of resin used for caries infiltration after exposure to different staining solutions Operative Dentistry 2014; 39(4): 433-440.

26. Torres CRG, Rosa PCF, Ferreira NS, Borges AB. Effect of caries infiltration technique and fluoride therapy on microhardness of enamel carious lesions. Oper Dent. 2012; 37: 363-9.

27. Paris S, Schwendicke F, Seddig S, Müller WD, Dörfer C, Meyer-Lueckel H. Micro-hardness and mineral loss of enamel lesions after infiltration with various resins: Influence of infiltrant composition and application frequency in vitro. J Dent. 2013; 41: 543-8.

28. Hahnel S, Henrich A, Burgers R, Handel G, \& Rosentritt M (2010) Investigation of mechanical properties of modern dental composites after artificial aging for one year Operative Dentistry 35(4) 412-419.

29. Borges AF, Santos Jde S, Ramos CM, Ishikiriama SK, \& Shinohara MS. Effect of thermo-mechanical load cycling on silorane-based composite restorations. Dental Materials Journal 2012; 31(6): 1054-1059.

30. Ulrich I, Mueller J, Wolgin M, Frank W, Kielbassa AM. Tridimensional surface roughness analysis after resin infiltration of (deproteinized) natural subsurface carious lesions. Clin Oral Investig 2015; 19(6): 1473-83.

31. Arnold WH, Meyer A-K, Naumova EA. Surface Roughness of Initial Enamel Caries Lesions in Human Teeth After Resin Infiltration. The Open Dentistry Journal, 2016; 10: 505-515.

32. Takashino N, Nakashima S, Shimada Y, Tagami J, Sumi Y. Effect of thermal cyclic stress on acid resistance of resininfiltrated incipient enamel lesions in vitro. Dental materials journal 2016; 35: 425-31.

33. Kielbassa AM, Ulrich I, Treven L, Mueller J. An updated review on the resin infiltration technique of incipient proximal enamel lesions. Med Evol 2010; 16: 3-15.

34. Leland A; Akyalcin S; English JD; Tufekci E; Paravina R. Evaluation of staining and color changes of a resin infiltration system. Angle Orthod. 2016; 86: 900-904. 